\title{
Intelligent Home Using Internet of Things
}

\author{
Zeba Sheikh, Abhishek Kommera, Sana Quazi \\ (Student, Dept. Of CSE, Ballarpur Institute of Technology, Gondwana University, MS, India) \\ (Assistant Professor,Dept. of CSE, Ballarpur Institute of Technology, Gondwana University, MS,India) \\ (Student, Dept. Of CSE, Ballarpur Institute of Technology, Gondwana University, MS, India)
}

\begin{abstract}
Intelligent home minimizes user's involvement in monitoring home and controlling home appliances with the help of Internet of Things. This paper proposes the advancement of an intelligent home application by integrating Internet of things (IOT) with web services and cloud computing. Our approach is to focus on entrenching intelligence into sensors using software and network connectivity. It facilitates interactions with smart things using cloud services. Our approach is more feasible and efficient i.e. it measures the home conditions and monitors the home appliances and controls the Home access.
\end{abstract}

Keywords: Internet of Things, Cloud, sensors, Intelligent home.

\section{Introduction}

Intelligent home is nothing but the automated home which is a collection of services and technology for increasing the quality of living. These services allow home to be automated. Home automation is exactly what it sounds like automating the ability of home objects to control the atmosphere or environment of home. Intelligent home filled with connected products are loaded with possibilities to make our lives easier, more convenient and more comfortable. Internet is a worldwide network of interconnected computer networks, based on standard communication protocol (TCP/IP). Things are the physical objects. These physical objects collect useful data with the help of various existing technologies and then autonomously flow the data between the other physical objects. Now by combining the Internet to the things a thriving technology has arrived into the hitech world. Internet of Things (IOT) links the object of real world with the digital world. Internet of Things is the internetworking of physical devices, abode, vehicles, buildings, and other objects-embedded with electronics, software, sensors, and network connectivity that enables these objects to collect and exchange data. We can describe this scenario in which object or people provided with unique identifiers and ability to transfer data over the network without requirement of human-to-human or human-to-computer interaction. Typically, IOT is expected to offer advance connectivity of devices, systems, and services that goes beyond machine-tomachine communications and covers a variety of protocols, domains, and applications. IOT represents a general concept for the ability of network devices to sense and collect data from the world around us and then share the data across the internet where it can be processed and utilized for various interesting purposes.

\section{System Architecture}

The figure shows system architecture for Intelligent Home which is consists of the above following components Our system consists of:

Hardware Used:

1. PIR Sensor

2. Motion sensor

3. Soil Moisture Sensor

4. Temperature Sensor

5. ESP8266-Standalone module

6. Relay Circuit

Technology and Platform Used:

1. IOT Technology.

2. ThingSpeak

3. Ubidots

PIR SENSOR: A Passive Infrared Sensor is an electronic sensor that measures infrared (IR) light radiating from objects in its field of view. They are most often used in PIR-based motion detectors.

The term Passive in this instance refers to the fact that PIR device do not generate or radiate any energy for detection purposes. They work entirely by detecting the energy given off by other objects. 
MOTION SENSOR: A motion sensor or detector is a device that detects moving objects, particularly people. Such a device is often integrated as a component of a system that automatically performs a task or alerts a user of motion in an area. They form a vital component of security, automated lighting control, home control, energy efficiency and other useful systems.

SOIL MOISTURE SENSOR: Soil Moisture Sensor measures the volumetric water content in soil.

Since the direct gravimetric measurements of free soil moisture requires removing, drying and weighting of a sample. Soil Moisture sensor measures the volumetric water content indirectly by using some other property of the soil.

TEMPERATURE SENSOR: It is a low voltage, precision centigrade sensor. It provides a voltage output that is linearly proportional to the Celsius temperature. It also doesn't require any external calibration to provide typical accuracies of $\pm 1^{\circ} \mathrm{C}$ at $+25^{\circ} \mathrm{C}$ and $\pm 2^{\circ} \mathrm{C}$ over the $-40^{\circ} \mathrm{C}$ to $+125^{\circ} \mathrm{C}$ temperature range.

ESP8266 STANDALONE MODULE: ESP8266 offers a complete and self-contained Wi-Fi networking solution, allowing it to either host the application or to offload all Wi-Fi networking functions from another application processor. When ESP8266 hosts the application, and when it is the only application processor in the device, it is able to boot up directly from an external flash. It has integrated cache to improve the performance of the system in such applications, and to minimize the memory requirements

RELAY: A relay is an electrically operated switch. Many relays use an electromagnet to mechanically operate a switch; Relays are used where it is necessary to control a circuit by a separate low-power signal, or where several circuits must be controlled by one signal. The advantage of relays is that it takes a relatively small amount of power to operate the relay coil, but the relay itself can be used to control motors, heaters, lamps or AC circuits which themselves can draw a lot more electrical power. The electro-mechanical relay is an output device (actuator) which comes in a whole host of shapes, sizes and designs, and has many uses and applications in electronic circuits.

INTERNET OF THINGS: Internet of Things is essentially an architectural framework which allows integration and data exchange between the physical world and computer systems over existing network infrastructure. Extending the current Internet and providing connection, communication, and inter-networking between devices and physical objects, or "Things," is a growing trend that is often referred to as the Internet of Things. "The technologies and solutions that enable integration of real world data and services into the current information networking technologies are often described under the umbrella term of the Internet of Things (IoT)"

THINGSPEAK: It is an open source Internet of Things application and also an API that used to store and retrieve the information from any entity by using the HTTP protocol via the web or LAN. Thing Speak allows the invention of sensor logging applications, location tracking applications and social network of entity by updating the status. ThingSpeak permits consolidation of various devices and application which can upload the data over the internet.

UBIDOTS: Ubidots offers a platform for developers that enable them to easily capture sensor data and turn it into useful information. Use the Ubidots platform to send data to the cloud from any Internet-enabled device.

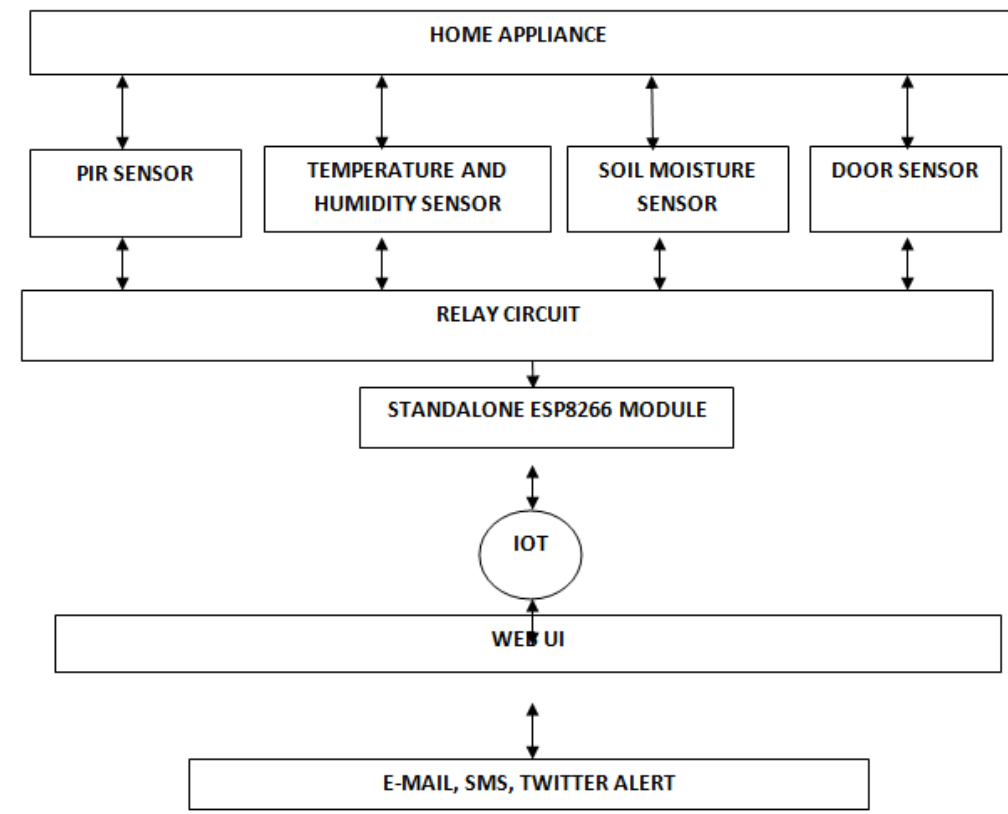

FIGURE: System Architecture of Intelligent Home 


\section{Working}

The working of our project is that we are using different sensors like PIR sensor for Intruder detection, Soil Moisture Sensor for Soil moisture, DHT11 for temperature and humidity. All sensors will individually connect to ESP8266 which is a standalone module with inbuilt Wi-Fi. All sensors connected to Wi-Fi will continuously monitor the data and send the data onto the cloud. Cloud will observe it and whenever it reaches to the certain threshold value, it will send an alert message to the user. The user can login by using his login and perform his action. The working principle of the intelligent home is wireless network technology i.e. IOT and home connected to each terminal and by use of this terminal can realize intelligent operation. After people consciously remote control, manual trigger corresponding emission intelligent equipment to recover the control command.

\section{Modules \& Results}

Intruder detection Using Passive Infrared Sensor:

For Intruder Detection we have used PIR Sensor. A PIR sensor for Intruder detection is used to sense movement of people, animals, or other objects. PIR Sensor will be fixed in the house and which will continuously sense the motion in the house. If the Sensor senses the unauthorized motion, it will update it on the Cloud. Cloud will send an alert message to the user.

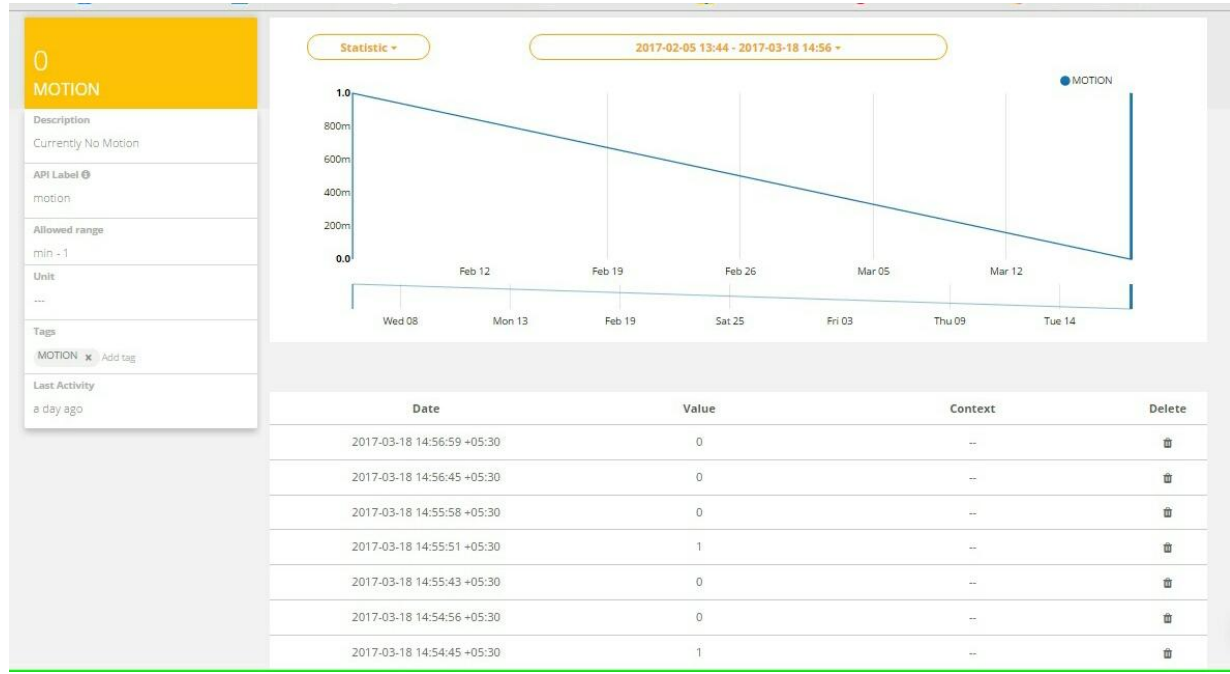

Figure 2: Graph showing the different motion sense by PIR Sensor.

Moisture Detection of Soil using Soil Moisture:

Here we have used Soil Moisture Sensor. Soil Moisture Sensor will monitor the moisture level in the soil of the garden. After sensing the moisture level of the soil, it will be updated on the Cloud whenever the moisture level of the soil reaches to the certain threshold it will alert it to the user.

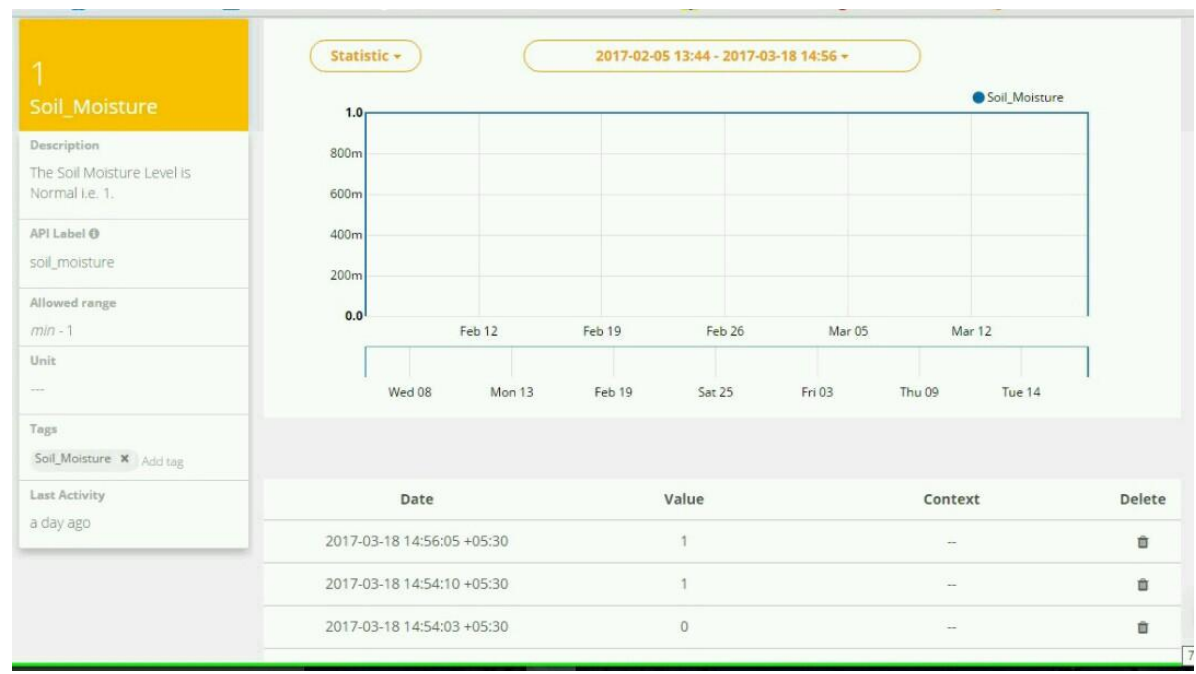

Graph showing the moisture level of Soil is Normal i.e. 1. 
Temperature \& Humidity Detection using DHT11:

For temperature and humidity we have used DHT11. The DHT11 is a basic, ultra low-cost digital temperature and humidity sensor. It uses a capacitive humidity sensor and a thermistor to measure the surrounding air, and spits out a digital signal on the data pin. After every 2 seconds data will b updated on the cloud. It is simple to use.

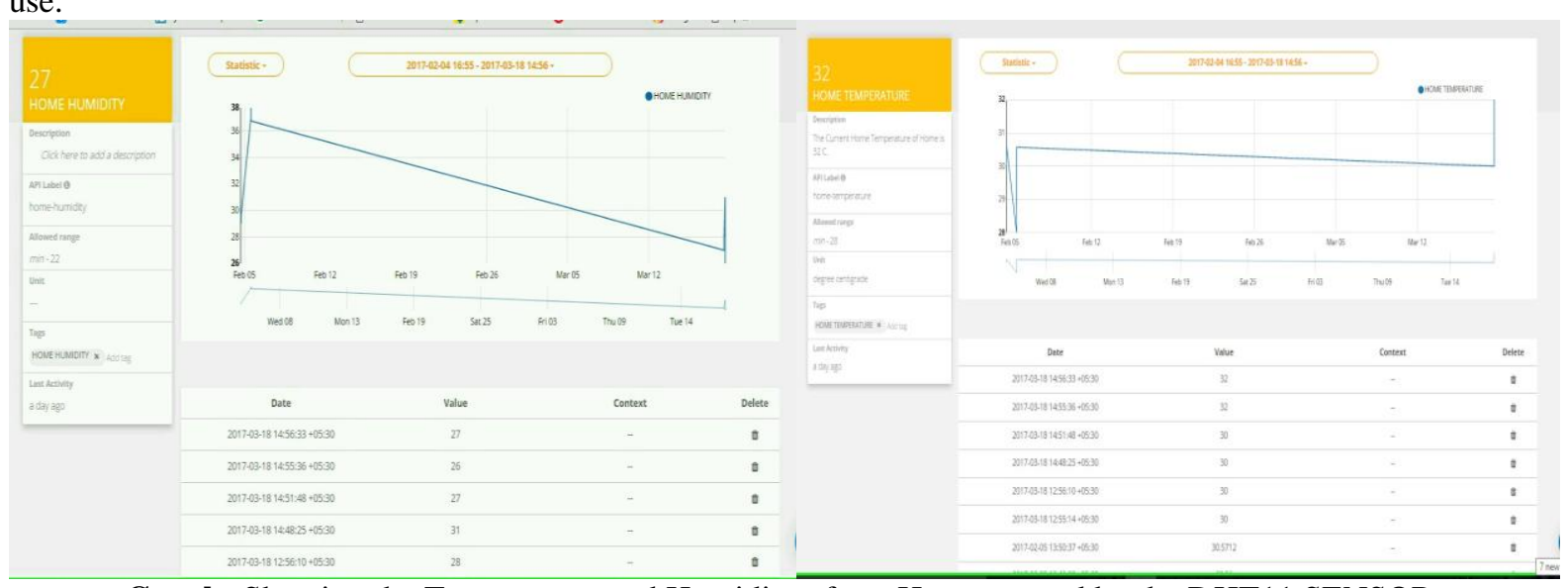

Graph: Showing the Temperature and Humidity of our House sensed by the DHT11 SENSOR.

Monitoring Security of Main Door Using Open/Close sensor:

For this module we have used Open/Close sensor. This Sensor maximizes both security and fleet efficiency by giving you trailer status updates based on door open/close events. Whenever it detects unauthorized access to your House yyou'll be alerted when the door sneaks. The sensor will continuously sense the data and it will be updated on cloud. Cloud will give an alert to the user.

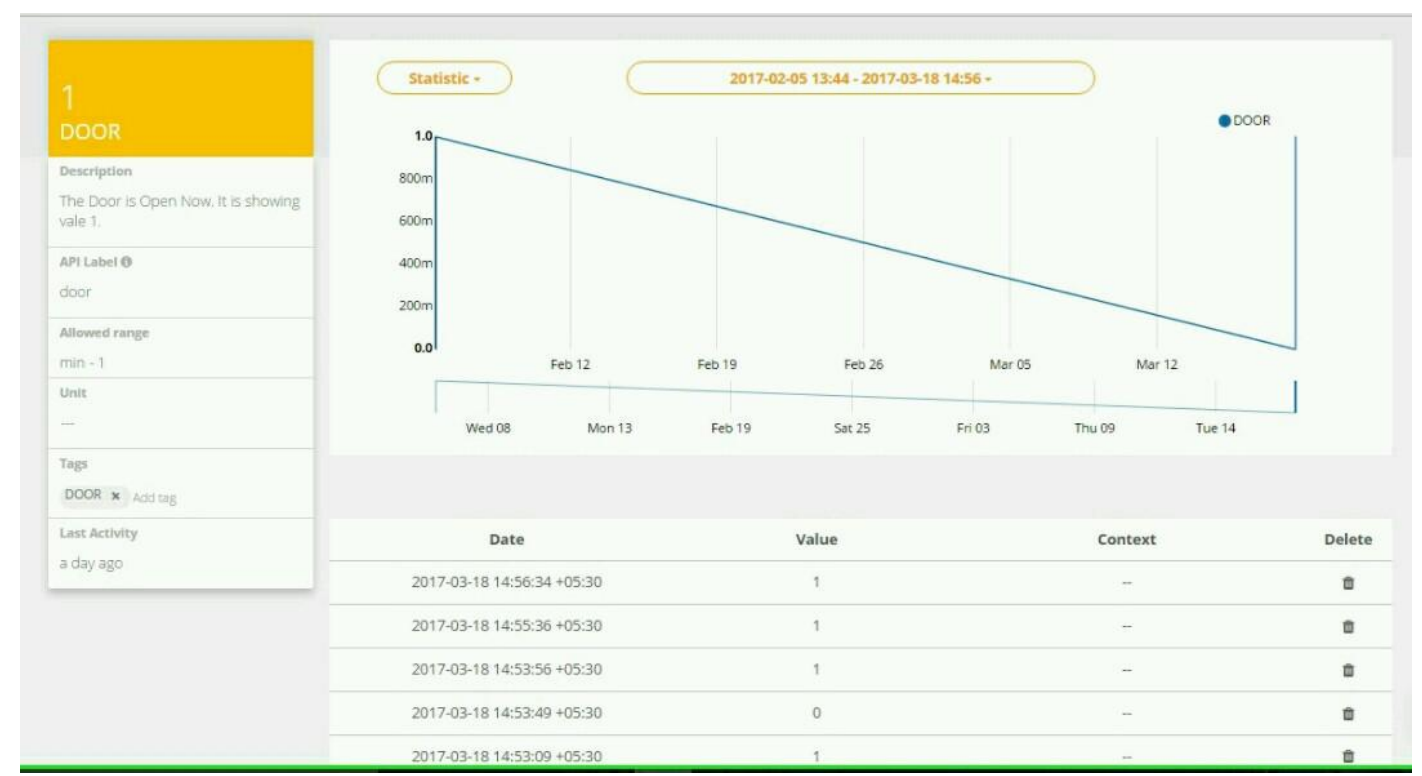

Graph showing the closing /opening of Main Door of House.

\section{Future of IOT}

The future of IoT is wide, millions and billions number of devices are connected to the internet. In 2014 near about 14.4 billion devices were connected to it but in future its predicted that it will be 50 billion or more than that will be connected to internet. Day by day the popularity of internet is increasing with the less involvement of human. People want everything to be "smart", Smart Home, Smart Phones, Smart Appliances, Smart Autos, Smart Cities, connected cars and many more from this some are already in our lives today and some will be come in our future. 


\section{INTERNET OF THINGS}

Number of Connected Devices Worldwide

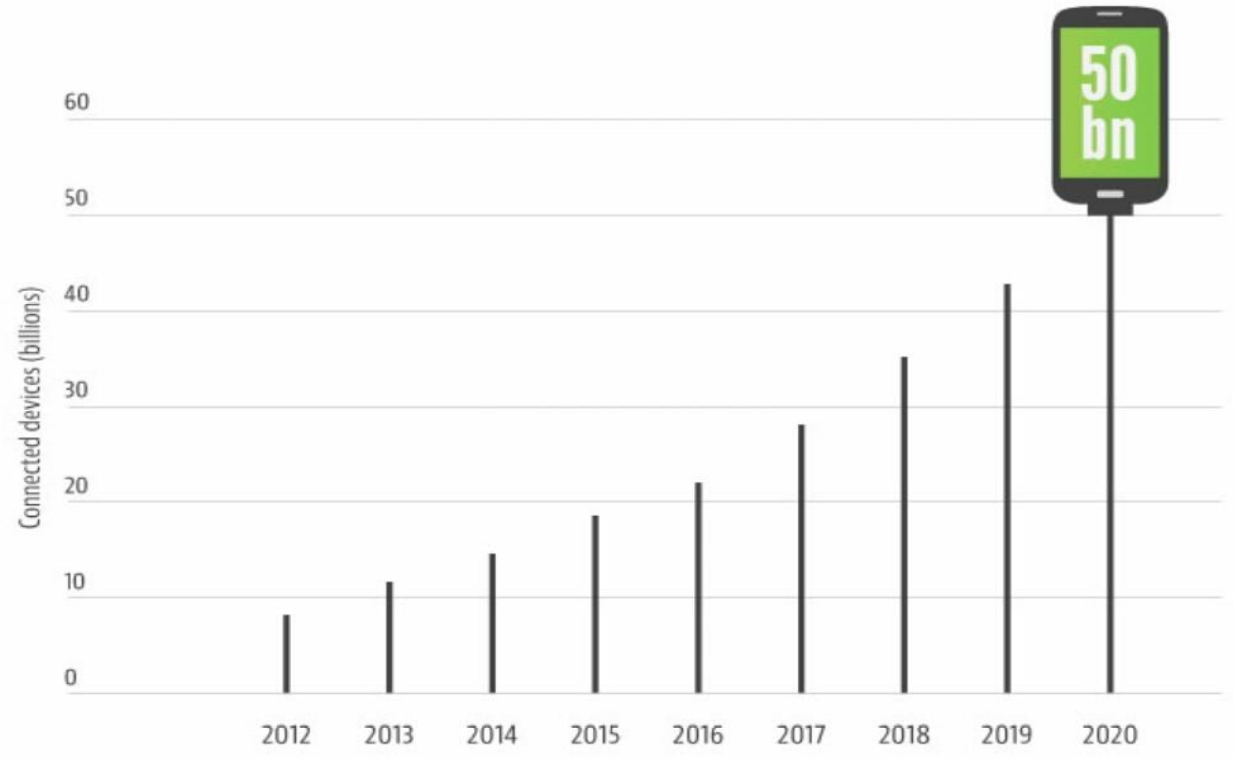

Graph: Showing the Future of Connected Devices

\section{Conclusion}

In our project we have used different technologies and hardware to integrate the sensors to the cloud. We have implemented more flexible architecture for integrating sensors to cloud using web services. We have also used ESP8266 which is a standalone module and has inbuilt Wi-Fi shield. ESP8266 is capable of hosting and application or offloading all Wi-Fi networking functions from another application processor. It makes more flexible and cost effective.

\section{Journal Papers:}

\section{Reference}

[1]. Diksha Jizilwar, Abhishek Kommera, Ashwini Gaddalwar, A Review on Intelligent Home Using Internet of Things, International Journal of Innovative Research in Computer and Communication Engineering, Vol. 5, Issue 1, January 2017

\section{Links}

[2]. https://www.programmableweb.com/api/ubidots

[3]. https://en.wikipedia.org/wiki/Internet_of_things

[4]. https://www.rs-online.com/designspark/thingspeak-a-free-internet-of-things-iot-platform http://www.ebay.com.au/itm/DHT11TEMPERATURE-AND-RELATIVE-HUMIDITY-SENSOR-TRANSDUCER-MODULE-/131606921333

[5]. http://www.skybitz.com/Portals/0/Documents/DoorSensor-Description.pdf

[6]. https://en.wikipedia.org/wiki/Passive_infrared_sensor

[7]. https://en.m.wikipedia.org/wiki/Internet_of_things

[8]. www.electronics-tutorials.ws/blog/relay-switch-circuit.html

[9]. https://www.sparkfun.com/products/10988

[10]. www.google.co.in/search?client=ms-opera-mini-android\&channel=new\&q=what + is + ubidots\&sa $=X \& v e d=0$ ahUKEwiPjYvEpDSAhXG7yYKHfEcBoEQ1QIIJCgA 